\title{
Revisión sobre el rol del profesional de relaciones públicas
}

\author{
Mónica Arzuaga Williams \\ Universidad de Málaga, España, y Universidad Católica del Uruguay, \\ Uruguay, Montevideo, Departamento de Comunicación.
}

marzuaga@ucu.edu.uy

Recibido: 20 de mayo de 2017. Aceptado: 28 de septiembre de 2017.

\section{Resumen}

Este artículo parte de los enfoques profesionales en los estudios de las relaciones públicas y da cuenta de las diferentes tipologías y formas de concebir el rol profesional, desde las primeras investigaciones sobre este campo disciplinar, con los estudios de Glen M. Broom y David M. Dozier en la década de 1980, hasta la actualidad, en que los cambios tecnológicos y el contexto mundial generan nuevas expectativas para el desempeño de este rol. La revisión se enmarcó en las publicaciones científicas de la colección principal de Web of Science que fueron clasificadas según su enfoque fuera sobre el desempeño del rol de los profesionales, sobre la situación en la que se desempeña el rol, o sobre la dinámica de los roles profesionales. En este trabajo se concluye que la mayoría de las tipologías sobre el rol del profesional de las relaciones públicas se basa en las declaraciones de los profesionales sobre las actividades desempeñadas y sobre las funciones o aportes del rol a las organizaciones. Entre los asuntos que atiende la literatura sobre el rol del profesional de las relaciones públicas predominan: las tensiones entre el rol del profesional de las relaciones públicas y el periodista; la discriminación según el sexo del profesional; las propuestas de tipologías de rol, y la descripción de las competencias y habilidades que se requieren para el desempeño del rol.

Palabras clave: relaciones públicas, comunicación organizacional, rol profesional, tipología de roles.

\section{Reviewing the role of public relations professionals}

\section{Abstract}

Taking professional approaches in public relations studies as a starting point, this article tracks the many ways in which this professional role has been perceived and identifies its different typologies, beginning with the first investigations into the field, carried out by Glen M. Broom and David M. Dozier in the 1980s, and then moving into the present day, when transformations in technology and in the international context are generating new expectations about how public relations should be performed. Our review centered on scientific publications from the Web of Science's main collection. These publications were classified depending on whether their focus was on the performance of the role of professionals, on the situation in which this role is performed, or on the dy- 
namics of professional roles. We conclude that most typologies of the role of public relations professionals are based on declarations by these professionals regarding their activities and how their role contributes to or operates within organizations. Among the topics found in the relevant literature, the most prominent are: the tensions between the role of public relations professionals and journalists, discrimination based on gender, role typology proposals, and the description of the competencies and skills required for the role. Keywords: public relations, communicational organizational, professional role, role typology.

\section{Revisão sobre o rol do profissional de relações públicas}

\section{Resumo}

Este artigo parte das abordagens profissionais nos estudos das relações públicas e apresenta as diferentes tipologias e formas de conceber o rol profissional, desde as primeiras pesquisas sobre este campo disciplinar, com os estudos de Glen M. Broom e David M. Dozier na década de 1980, até a atualidade, em que as mudanças tecnológicas e o contexto mundial geram novas expectativas para desempenho deste rol. A revisão foi enquadrada nas publicações científicas da coleção principal de Web of Science que foram classificadas segundo qual fosse sua abordagem: sobre o desempenho do rol dos profissionais, sobre a situação na que se desempenha o rol, ou sobre a dinâmica dos roles profissionais. Neste trabalho se conclui que a maioria das tipologias sobre o rol do profissional das relações públicas está baseada nas declarações dos profissionais sobre as atividades desempenhadas e sobre as funções ou contribuições do rol para as organizações. Entre os assuntos que a literatura observa sobre o rol do profissional das relações públicas, predominam: as tensões entre o rol do profissional das relações públicas e o jornalista; a discriminação segundo o sexo do profissional; as propostas de tipologias de rol, y a descrição das competências e habilidades que são requeridas para o desempenho do rol.

Palavras chave: relações públicas, comunicação organizacional, rol profissional, tipologia de roles.

\section{La perspectiva profesional en los estudios de las relaciones públicas}

A pesar del gran crecimiento de programas académicos de formación universitaria de grado y posgrado en relaciones públicas $^{1}$, el desarrollo de publicaciones científicas especializadas y la creación de asociaciones profesionales y académicas muy activas y consolidadas, distintas revisiones de la literatura científica muestran escasa presencia de artículos teóricos o epistemológicos en relaciones públicas (Botan \& Taylor, 2004; Ferguson, 1984). Desde sus inicios, los estudios en este campo se han nutrido de marcos conceptuales de otras disciplinas de las ciencias sociales, en especial de la comunicación, la sociología, las ciencias políticas y la administración. Esta escasa contribución teórica propia — generada por y desde la misma disciplina-ha provocado

\footnotetext{
En este trabajo se utiliza el término relaciones públicas porque predomina en la literatura indizada en las bases de datos de publicaciones científicas. En las revisiones se incluyó otro término de búsqueda: comunicación organizacional, más complejo y abarcador que el primero. Además, en la búsqueda no se excluyeron los resultados con otros términos de uso dentro de la disciplina, tales como comunicación corporativa, gestión de la comunicación, Dircom, entre otros. En este artículo no se discute la demarcación epistemológica entre estos términos.
} 
ambigüedades o confusiones entre las relaciones públicas entendidas como objeto de estudio, como práctica profesional y como disciplina científica (Hutton, 1999; Ulloa, Apolo, \& Villalobos, 2015; Xifra, 2011).

La más clara y sistemática generación de conocimiento en los estudios sobre relaciones públicas surge de la investigación empírica sobre sus funciones, las prácticas y las técnicas que aplican o deberían aplicar los profesionales. La prevalencia de las perspectivas profesionales frente a las teóricas es resultado del origen y naturaleza de una disciplina que - aunque integra el campo de las ciencias sociales- prioriza la práctica profesional y el aporte que esta hace a los objetivos organizacionales, es decir, sus aspectos funcionales. Sumadas a esta característica, las perspectivas profesionales de la disciplina han tenido, desde mediados del siglo pasado, un fuerte desarrollo debido al interés del campo laboral y del mercado hacia sus prácticas y resultados, así como también por una extensiva implantación de la formación universitaria en relaciones públicas en el mundo occidental (Castillo Esparcia, 2009).

En las dos últimas décadas del siglo XX surge una articulación formal del pensamiento sobre la práctica profesional de las relaciones públicas, con base en la función estratégica de la profesión y con aportes sobre los modelos de dirección y gestión de las unidades de relaciones públicas en las organizaciones. Esta articulación formal se da a partir de los Estudios de la Excelencia liderados por James E. Grunig (Grunig, 1992; Grunig \& Huang, 2000; Grunig \& Hunt, 1984), que se pueden considerar el paradigma dominante en los estudios de las relaciones públicas. Además de estimular la institucionalización de la profesión, la propuesta de los Estudios de la Excelencia marcó con énfasis la orientación curricular en las universidades y también la agenda de la investigación empírica y la agenda de la divulgación en relaciones públicas en gran parte del mundo (Botan \& Taylor, 2004).

Más recientemente, modelos derivados de las teorías de la comunicación, de la administración y de las ciencias políticas — por ejemplo, las perspectivas de las relaciones públicas en la gestión de asuntos públicos y el manejo de crisis, entre varias- han enriquecido los estudios sobre el profesional de Relaciones Publicas (Botan \& Taylor, 2004). Estos, sin embargo, aún no han logrado acercarse al predominio de los modelos derivados de los Estudios de la Excelencia.

Es recién a principios del siglo XXI cuando se fortalece el debate entre dos corrientes de pensamiento con distintos paradigmas y tradiciones: los Estudios de la Excelencia y la Teoría Crítica (L'Etang, 2009). Este debate, que pretende consolidar epistemológicamente los estudios en el campo disciplinar, seguramente derivará en modelos alternativos para el estudio del rol del profesional de las relaciones públicas. 


\section{Tipologías del rol del profesional de relaciones públicas}

Dentro de las perspectivas profesionales del estudio de una disciplina, la preocupación por la definición y clasificación del rol profesional ocupa gran parte de la investigación y reflexión. En este sentido, se presentan a continuación los modelos de estudio del rol del profesional de las relaciones públicas con sus propuestas sobre tipologías de roles.

La primera aproximación a una tipología de roles del profesional de relaciones públicas fue la publicada en 1979 por Kalman B. Druck y Ray E. Hiebert (citado en Broom \& Dozier, 1986, pp. 37-38) que establecían niveles de desarrollo de la carrera de los profesionales miembros de la Public Relations Society of America (PRSA). En esta clasificación, los autores definen cuatro niveles de carrera según la antigüedad, la autoridad o el salario, entre otras variables, y los asocian a cuatro roles: profesional senior, gerente profesional, personal profesional, profesional principiante (senior professional, professional manager, staff professionaly beginning professional) $)^{2}$.

El mismo año, Mary Ann Ferguson (citada por Broom \& Dozier, 1986, p. 40) publica un relevamiento sobre las tareas que desempeñan los profesionales miembros de la PRSA y elabora cuatro clústeres, que denomina roles mayores, según el tipo de contenido de cada rol: gerente solucionador de problemas, comunicador técnico-periodista, investigador, gerente de personal (problem-solving manager, journalist-technical communicator, researcher y staff manager).

La debilidad de estos dos modelos radica en que las categorías propuestas no son excluyentes y son de diferente naturaleza. La definición de senior y beggining corresponden a etapas de una carrera basada en la experiencia y responsabilidad del profesional; las categorías manager y staff responden a funciones administrativas y su ubicación en la estructura organizacional: la primera en nivel jerárquico con responsabilidad en la toma de decisión y la segunda en áreas de apoyo sin personal a cargo. Por otra parte, journalist y communicator corresponden a dos profesiones en sí mismas. Y las categorías de problem-solving y researcher corresponden a competencias y actividades, o conjunto de actividades, que pueden ser desarrolladas por todas las otras categorías. En definitiva, estas dos propuestas mezclan las actividades o tareas que realiza el profesional con las competencias o habilidades que requiere para realizarlas, con la finalidad o la función para la cual las realiza, la ubicación que tiene el profesional en la estructura organizacional y el nivel de desarrollo de la carrera.

\footnotetext{
Se incluyen siempre las denominaciones en inglés, porque existen diferentes usos de estos términos en el idioma español. Por ejemplo, el término manager se puede traducir como gerente, director, gestor, administrador, y cada término en español responde, a su vez, a diferentes tradiciones de escuelas de administración y diferentes usos, según el tipo de organización (pública, privada, con o sin fines de lucro, etcétera).
} 
Con base en el modelo de consultoría de procesos creado por Chris Argyris (1961) y desarrollado por Edgar Schein (1969), y con el aporte de Scott M. Cutlip y Allen H. Center (1971), los investigadores Broom y Smith (1979) realizaron un estudio de caso con diseño experimental y a partir de los resultados proponen una clasificación para categorizar los tipos de roles de los profesionales de las relaciones públicas dedicados a la consultoría. Esta clasificación se focaliza en cómo los diferentes roles de consultoría afectan la relación cliente-profesional y cuáles son las percepciones mutuas y las evaluaciones, tanto de clientes como de los consultores, sobre los resultados y contribuciones de la consultoría. En este sentido, se trata del primer modelo de roles profesionales de las relaciones públicas que integra las expectativas propias del actuante del rol (el consultor) y las expectativas de quienes demandan y evalúan el rol (los clientes del consultor). Los autores concluyen en una tipología de cinco roles: prescriptor experto, facilitador de procesos de comunicación, facilitador de procesos de resolución de problemas, proveedor de servicios técnicos, legitimador de aceptación (expert prescriber, communication process facilitator, problem-solving process facilitator, technical services provider y acceptant legitimizer). Esta última categoría luego fue descartada en otros estudios.

Broom y Dozier en 1986 - a partir de un trabajo de 1983 de Dozier - realizan una nueva encuesta sobre la misma muestra utilizada por Druck y Hiebert. A partir de los resultados sintetizan la tipología de roles propuesta por Broom y Smith, y afirman que los roles se pueden reducir a dos: gerente (manager) - que incluye a los tres primeros de la clasificación original-y técnico (technician). Esta tipología, que toma como modelo conceptual de análisis la teoría de los roles organizacionales propuesta por Katz y Kahn (citado en Dozier \& Broom, 2006, pp. 138-143), es el modelo que ha tenido mayor influencia en la literatura sobre el rol del profesional de las relaciones públicas. Se trata de un modelo de roles predominantes dentro de la dicotomía de gerente de comunicación (communication manager) y técnico en comunicación (communication technician) que es construido a partir de la frecuencia de desempeño de determinadas acciones y actividades de los profesionales de las relaciones públicas (Dozier \& Broom, 2006, pp. 140-141).

El primero, el de communication manager ${ }^{3}$, es el rol que involucra al profesional de las relaciones públicas en la coalición dominante de la organización. Este lleva adelante procesos sistemáticos de planificación, sirve como catalizador de los procesos de decisión, facilita la comunicación entre la coalición dominante y los públicos, y genera información sobre reacciones a las políticas, procedimientos y acciones. El segundo, el

3 Este término, traducido como director de comunicaciones, es retomado y propuesto como denominador del rol profesional por parte de la Asociación de Directivos de Comunicación (DIRCOM). 
de communication technician, es el del profesional de las relaciones públicas que, según los autores, presenta actividades repetitivas más fácilmente estandarizables.

Más adelante en el tiempo, como revisión de su propio modelo, Dozier y Broom (1995) sugieren que es necesario explorar el perfil creativo artístico que también se observa en el desempeño del rol profesional de las relaciones públicas y que no se expresa claramente en las actividades propuestas para su modelo dicotómico.

De cualquier modo, la dicotomía propuesta parece aplicarse con claridad a roles definidos por la naturaleza de las funciones del profesional de las relaciones públicas según el nivel jerárquico que ocupa dentro de una organización, es decir, según cuán cerca se encuentra de la toma de decisiones clave en una organización. Sin embargo, esta clasificación no es suficientemente exhaustiva para describir el rol profesional con independencia de su nivel funcional en la organización.

Este modelo ha sido aplicado en diferentes países, en ocasiones, con algunos ajustes como la modificación de la batería de preguntas, la aplicación del campo a diferentes sujetos de estudio, diversos análisis estadísticos en los resultados, etcétera. Su valor más significativo es que ha generado una nutrida agenda de investigación con numerosos autores que aplican o discuten algunas de las propuestas del modelo. Pero la mayoría de los autores coincide con que una de las variables clave para diferenciar los roles de los profesionales de las relaciones públicas es la participación en la toma de decisiones estratégicas en la organización.

Algunos autores cuestionan que el modelo sea dicotómico: entienden que la excesiva simplificación no permite acercarse a la compleja realidad. Greg Leichty y Jeff Springston (1993), con base en una encuesta a profesionales de una asociación de Alabama, plantean que las categorías propuestas por Broom y Dozier no son mutuamente excluyentes, porque un mismo profesional puede desarrollar actividades que corresponden a ambos roles, según el tipo de públicos con los que se relacione y el tipo de relaciones que establezca con cada uno de ellos.

Leichty y Springston (1993) proponen un modelo de cuatro roles: gerentes, internos, externos, generalistas (managers, internals, externals y genaralists) y un rol residual. Cada uno de ellos presenta un diferente nivel de prioridad sobre ocho factores que representan las diversas actividades de los profesionales de las relaciones públicas. Porter \& Sallot (2003), continuando las investigaciones empíricas de Leichty y Springston, realizan una encuesta autoadministrada a profesionales y proponen modificar el rol de generalista por el de técnico (technician) y agregan un noveno factor y otras actividades.

Por otra parte, Danny Moss y un equipo de investigadores (Moss, Warnaby \& Newman, 2000; Moss \& Green, 2002; DeSanto \& Moss, 2005; Moss, Newman, \& DeSanto, 2005; DeSanto, Moss, \& Newman, 2007) realizan una serie de estudios en el 
Reino Unido y en los Estados Unidos sobre los contenidos del rol gerencial (managerial role) en relaciones públicasy comunicación organizacional entre profesionales de nivel jerárquico alto de organizaciones de diferente tipo, tamaño y sector.

Estos autores sostienen que las tipologías que se han propuesto no pueden ser universales ni exhaustivas (Moss \& Green, 2002, p. 120), porque el desempeño de roles es contingente a varios factores, entre ellos, los propios de la organización: tipo, estructura, sector y entorno; los que dependen del estilo de liderazgo y dirección, como comprensión, visión y expectativas que la dirección tenga sobre la función y el valor de las relaciones públicas o la comunicación; los factores que refieren a la naturaleza de la función gerencial que desarrolle el profesional (Moss, Warnaby \& Newman, 2000, pp. 295-299); otros factores están determinados por el cargo y jerarquía, el tamaño de la unidad de comunicación, el nivel al que reporta el profesional, la participación en la toma de decisiones estratégicas que tenga el profesional, y, finalmente, también intervienen factores personales como las preferencias y competencias personales del profesional que desempeña el rol (DeSanto \& Moss, 2005, pp. 185-188).

A partir de sus investigaciones, los autores concluyen que el rol del profesional de las relaciones públicas o la comunicación organizacional, en su nivel gerencial directivo, muestra cinco dimensiones: asesor en políticas claves y estratégicas; monitor y evaluador; experto en gestión de asuntos claves; mediador y solucionador de problemas, y técnico de comunicaciones (key policy and strategy advisor, monitor and evaluator, issue management expert, troubleshooter problem-solver, communications technician).

Otros autores derivan los tipos de roles profesionales de las teorías de la comunicación. Por ejemplo, Betteke van Ruler (2004) define siete tipos ideales de roles para los profesionales de las relaciones públicas o la comunicación organizacional, según estén orientados a la función informacional - roles orientados a la distribución de mensajes, la representación unidireccional y la transmisión de información básicamente con significados denotativos-; a la función de persuasión — roles que buscan la sincronización de significados connotativos desde una posición de poder, como la creación de imagen, ejercer influencia-, o a la función comunicacional — roles basados en procesos como la interacción, la creación de acuerdos y consensos, la mediación y el diálogo para la creación de significado con los públicos. A estos roles los identifica con metáforas: el pregonero, el mayordomo, el policía de tránsito, el director de orquesta, el creador, el facilitador y el intuitivo o improvisado (the town crier, the steward, the traffic manager, the conductor, the creator, the facilitator, the seat-of-the-pants).

Van Ruler, Verčič, Butschi y Flodin (2001), en tanto, a partir de un estudio Delphi en 25 países europeos, proponen cuatro estilos de roles en función de los aportes que hace el profesional de las relaciones públicas o la comunicación organizacional a la organización: el reflexivo, el directivo, el operativo y el educativo. 
Finalmente, Anne Gregory (2015) sistematiza el relevamiento realizado entre 2011 y 2012 para el Melbourne Mandate ${ }^{4}$ e identifica tres roles para los profesionales de las relaciones públicas y la comunicación organizacional, que son críticos para las organizaciones y se proponen como roles aspiracionales, es decir, a los que debería orientarse el profesional. Estos son: definir y mantener los valores y la identidad (character) organizacional; construir una cultura de escucha y compromiso; instalar la responsabilidad social, organizacional, individual y profesional.

A continuación, se presenta un esquema sintético de estos modelos de tipologías en el que se señalan para cada uno: los autores más significativos, el año de la primera publicación del modelo, la base empírica de la que surgen las evidencias y conclusiones del modelo, el enfoque con el que definen el rol y la tipología de roles propuesta. Como se observa en la tabla, la mayoría de las tipologías se basan en las actividades que desempeña el profesional y en las funciones o aportes que hace el profesional a la organización.

\section{Revisión sobre el rol del profesional de relaciones públicas}

Los siguientes apartados presentan el diseño y luego los resultados de una revisión $^{5}$ de la literatura científica accesible en la colección principal de Web of Science, que incluye las bases de Science Citation Index Expanded, Social Sciences Citation Index, Arts \& Humanities Citation Index, Conference Proceedings Citation Index y Emerging Sources Citation Index. Los contenidos no son representativos de toda la literatura científica y la literatura de divulgación en el campo, pero es una evidencia razonable de lo que es considerado como conocimiento al respecto entre pares evaluadores de la disciplina. Una futura ampliación de esta revisión se podría realizar en libros académicos de editoriales especializadas en el campo de las relaciones públicas y la comunicación organizacional, a modo de ejemplo, Routledge, Sage y Editorial UOC.

La primera búsqueda se realizó para identificar el universo de artículos cuyo tema refiere al campo disciplinar general de las relaciones públicas; tuvo el siguiente criterio de consulta TS = ("public relations" OR "organizational communication") y se obtuvieron 4. 094 resultados. Luego se refinó la búsqueda con el objetivo de delimitar, dentro de ese universo, los artículos científicos cuyo tema refiere a la práctica profesional de manera explícita. Para ello se aplicó el criterio de búsqueda TS= (("public relations" OR "organizational communication") AND (practitioner OR professional OR role)), y se obtuvieron 1.966 resultados.

\footnotetext{
Documento propuesto por la Global Alliance for Public Relations and Communication Management en ocasión del World Public Relations Forum de 2012 celebrado en Melbourne (Australia).

5 La última actualización de los datos estadísticos de la revisión bibliográfica se realizó el 20 de marzo de 2017.
} 


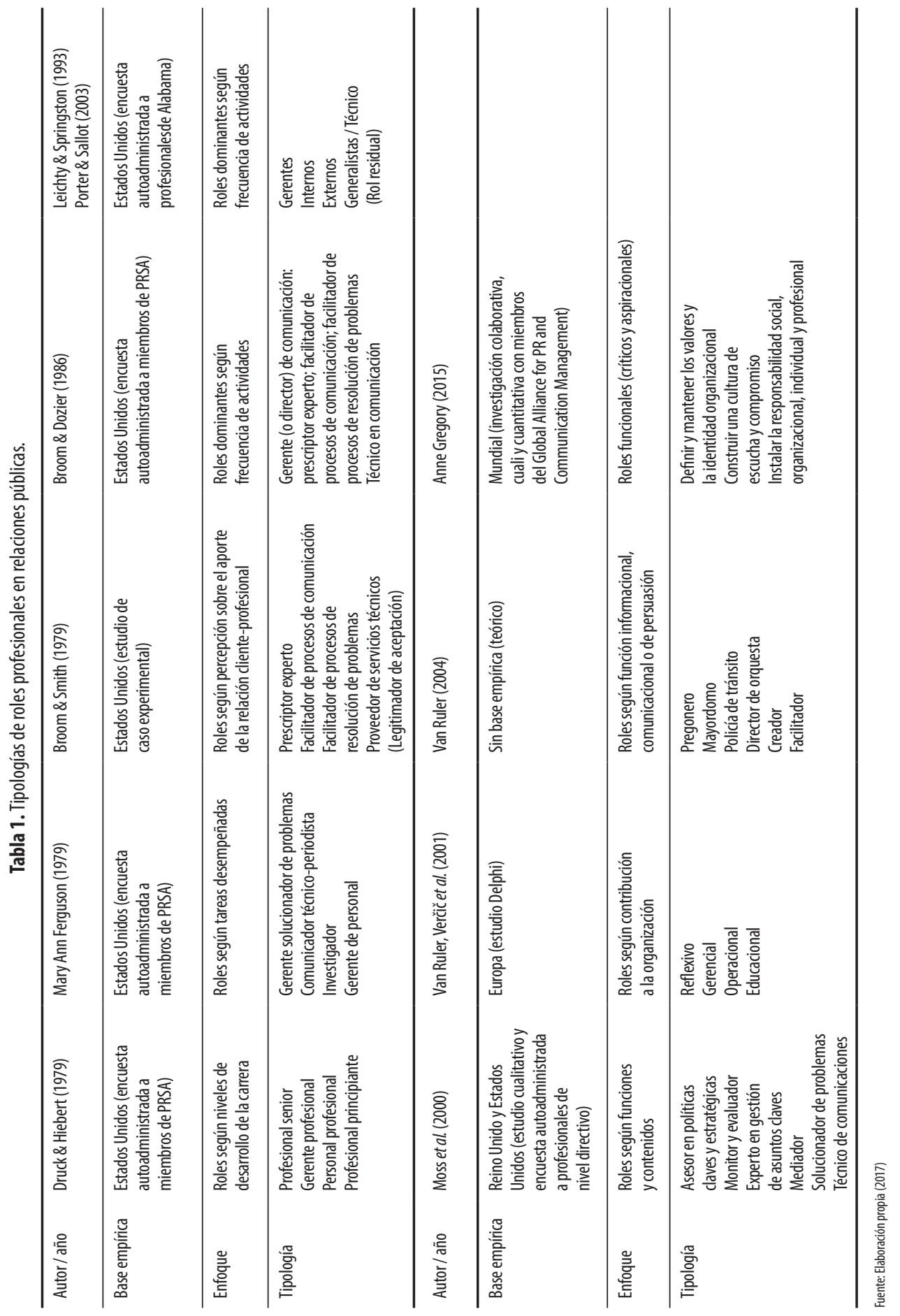


Es decir, el $48 \%$ de los artículos disponibles sobre relaciones públicas en la colección principal de Web of Science se refieren explícitamente a temáticas relativas a los aspectos profesionales. Esto evidencia, tal como se planteó en el primer apartado, el predominio de los enfoques profesionales por sobre los artículos teóricos, epistemológicos y metodológicos en la literatura sobre relaciones públicas.

Con el objetivo de ajustar la muestra para realizar el análisis de su contenido, se refinó el resultado de esta búsqueda para encontrara artículos sobre asuntos profesionales que tuvieran al menos un indicador fuerte, definido intencionalmente por sus autores, asociado al objeto de análisis: la presencia de algunos de los términos de búsqueda en el título. Para ello se aplicó el siguiente criterio TI= (("public relations" OR "organizational communication") AND (practitioner OR professional OR role)). En esta búsqueda se obtuvieron 224 registros.

Finalmente, luego de la revisión de títulos, palabras clave y resumen de cada artículo, se construyó una muestra de 112 artículos que cumplían con todos los requisitos de búsqueda: trataban como tema central en su desarrollo - y esto era expresado en su título, palabras clave o resumen - algún aspecto del rol profesional (identificado como professional o como practitioner, practicante) en relaciones públicas o comunicación organizacional.

Es razonable asumir que entre los artículos descartados pueda haber contenidos relativos al estudio del rol del profesional de las relaciones públicas, que figuren con otras clasificaciones en las bases científicas. No obstante, la muestra analizada es, sin lugar a dudas, el núcleo duro de contenido sobre la temática, ya que se trata de los artículos que han sido explícitamente clasificados como tales por sus autores, evaluadores e indizadores.

Algunos artículos desarrollan en sus contenidos más de un enfoque de estudio y, por lo tanto, podrían entrar en más de una de las categorías temáticas propuestas a continuación. En esos casos, se optó por clasificarlo según la categoría que figura de manera explícita en el resumen o en las palabras clave, es decir, la priorizada por el autor. Cada categoría temática se ilustra con algunos artículos referentes.

\section{Resultados de la revisión}

A continuación, se presenta un cuadro sintético y luego, el desarrollo de los resultados de la revisión de literatura clasificados según temática del artículo y agrupados según pertenezcan a distintas áreas de estudio sobre los roles: el desempeño del rol, la situación del rol, o la dinámica del rol. 
Tabla 2. Enfoques de los artículos sobre rol profesional en las relaciones públicas.

\begin{tabular}{lc}
\hline Enfoque & Porcentaje \\
\hline Sobre el desempeño del rol & 82,7 \\
Tensiones y conflictos de roles & 31,8 \\
Modelos y tipologías de roles & 30,9 \\
Contenidos de roles & 20,0 \\
Sobre la situación del rol & 9,1 \\
Sobre la dinámica del rol & 8,2 \\
\hline TOTAL & 100,0 \\
\hline
\end{tabular}

\section{Sobre el desempeño del rol del profesional de relaciones públicas}

Los estudios sobre el desempeño del rol buscan identificar los patrones en términos de tareas, actividades, contenidos o competencias de los roles y llegan a la construcción de tipologías. Además, describen y explican los conflictos entre diferentes expectativas y entre diferentes roles. Por lo general, estas investigaciones tienen diseños descriptivos y algunos correlacionales y siempre se centran en el actuante del rol a partir de sus percepciones, declaraciones sobre comportamientos, opiniones, etcétera.

Sin lugar a duda, este tipo de estudios predominan en la literatura: el $82,7 \%$ de los artículos de la muestra están directamente orientados en este sentido. Este volumen de artículos permite segmentar internamente el conjunto y observar que hay tres asuntos que destacan: el análisis de tensiones y conflictos entre roles $(31,8 \%$ del total de artículos de la muestra), la propuesta de modelos con tipologías de desempeño de roles (30,9\% del total de la muestra) y los estudios de contenidos, competencias y habilidades del rol profesional ( $20 \%$ del total de la muestra). A continuación, se detallan los enfoques dentro de cada segmento.

\section{Tensiones y conflictos de roles profesionales}

Casi la mitad de los artículos en este segmento tratan sobre la diferenciación, la integración y las tensiones entre las relaciones públicas y otros roles profesionales del campo de la comunicación.

Casi la totalidad de los artículos en este segmento se refiere a las tensiones entre los roles del periodista y el profesional de las relaciones públicas (Berkowitz \& Lee, 2004; Leichty \& Springston, 1996; Kim \& Bae, 2006; Mellado \& Hanusch, 2011; Neijins \& Smit, 2006). Se trata de una relación entre profesionales que, por sus funciones, tienen contactos muy frecuentes, intensos y muchas veces son interdepen- 
dientes entre sí. Pero, como destacan los autores, los periodistas y los profesionales de las relaciones públicas tienen finalidades diferentes y, en ocasiones, opuestas (Shin \& Cameron, 2004; Shin, Lee, \& Park, 2012; Verčič \& Colic, 2016).

Es así como en varios artículos se sostiene que el periodista se desempeña con base en las expectativas de los ciudadanos; en cambio, el desempeño del profesional de las relaciones públicas está orientado por las expectativas de clientes, empleadores y públicos. Muchos de los artículos destacan especialmente el conflicto deontológico de los profesionales que ejercen ambos roles en forma simultánea (Frohlich, Koch, \& Obermaier, 2013; Macnamara, 2016; Obermaier \& Koch, 2014).

Un pequeño número de artículos enfoca las diferencias y similitudes entre el rol de los profesionales de la publicidad y el de los profesionales de las relaciones públicas a partir de la era digital (Supa, 2016; Toledano, 2010); o las diferencias conceptuales entre los profesionales del marketing y los de las relaciones públicas (Ha \& Ferguson, 2015; Spicer, 1991).

La otra mitad de los artículos analizados en este segmento se centra en el conflicto en el sentido de discriminación, diferenciación e inequidad en el reconocimiento al desempeño del rol (Wolf, 2016; Chen, 2011). La mayoría de los artículos en este segmento hacen foco en la diferenciación o discriminación según el sexo del profesional de las relaciones públicas. Predominan los artículos que marcan la influencia que tiene el sexo del profesional en la construcción de las identidades, en el tipo de rol gerencial o técnico y en el reconocimiento y remuneración que obtiene. También se destacan los artículos sobre el conflicto entre la vida familiar y la laboral (Algren \& Eichhorn, 2007; Jiang \& Shen, 2013; Molleda \& Ferguson, 2004; Penninga \& Sweerserb, 2015; Sha \& Toth, 2005; Toth, 1988; Toth, Serini, Wright, \& Emig, 1998; Yaxley, 2013).

Algunos de los artículos atienden la discriminación étnica o cultural entre profesionales (Brown, White, \& Waymer, 2011; Wolf, 2016).

\section{Modelos y tipologías de roles profesionales}

Los artículos en este segmento de la muestra proponen o discuten modelos de rol profesional, tipologías de rol, o realizan aplicaciones de esos modelos y tipologías en países o en casos particulares, por ejemplo, entre los miembros de una asociación profesional o entre los profesionales vinculados a las grandes corporaciones.

Casi todos los artículos en este segmento proponen o asumen la naturaleza estratégica del rol del profesional de las relaciones públicas. También la mayoría de los artículos adscriben a los Estudios de la Excelencia o aplican el modelo de Broom y Dozier para tipificar los roles profesionales. 
A pesar del predominio del modelo propuesto por Broom y Dozier, uno de cada tres artículos en este segmento (28\% del total del segmento) plantea propuestas conceptuales diferentes de la dominante, discute ese modelo y lo modifica o le agregan nuevas categorías, en general en función de evidencia empírica. Entre estos autores se encuentran DeSanto \& Moss, 2005; DeSanto, Moss, \& Newman, 2007; Dodd, Brummette, \& Hazleton, 2015; Hutton, 1999; Le Roux, 2014; Leichty \& Springston, 1993, 1996; Li, Cropp, Sims, \& Jin, 2012; Mellado \& Barria, 2012; Moss \& Green, 2002; Moss, Newman, \& DeSanto, 2005; Moss, Warnaby \& Newman, 2000; Yeo \& Sriramesh, 2009.

Finalmente, algunos autores proponen modelos de roles o tipologías basados en marcos teóricos diferentes de los predominantes en la literatura, por ejemplo, Van Ruler, Verčič, Butschi, \& Flodin (2001, 2004); Van Ruler (2004), Gregory (2008, 2015).

\section{Contenidos del rol profesional}

El resto de los artículos que estudian el desempeño del rol de los profesionales de las relaciones públicas, tratan sobre los contenidos del rol, es decir, las competencias y habilidades que se requieren para el desempeño del rol profesional en el campo (Algren \& Eichhorn, 2007; Callison, Merle, \& Seltzer, 2014; Dozier, 1984, 1985; Napoli, Taylor, \& Powers, 1999; Todd, 2014; Vieira Jr \& Grantham, 2014).

En este conjunto de artículos se destacan los relativos a las nuevas competencias requeridas a partir de los cambios generados, en primer lugar, con internet y, más tarde, con la integración de las redes sociales en las estrategias de relaciones públicas (Alikilic \& Atabek, 2012; Lauzen, 1992; Lee, Sha, Dozier, et al., 2015; Moreno, Navarro, Tench, \& Zerfass, 2015; Porter \& Sallot, 2003; Sallot, Porter, \& Acosta-Alzuru, 2004).

Varios de los artículos exploran las funciones específicas o esperables del rol profesional en coyunturas particulares, por ejemplo, en períodos de crisis (Guth, 1995; Hu \& Pang, 2016), o en relación con procesos y modelos de la administración, por ejemplo, las competencias para atender la responsabilidad social empresarial o la calidad total (Kim \& Reber, 2015; Heath, Leth, \& Nathan, 1994).

\section{Sobre la situación del rol del profesional de relaciones públicas}

Los estudios sobre la situación del rol también se centran en los actuantes del rol, pero buscan identificar las variables que intervienen en su desempeño, por ejemplo, el tipo de organización: pública, privada, multinacional, con o sin fines de lucro; o cómo intervienen los procesos organizacionales de liderazgo, motivación o clima en el desempeño de un rol; también se ocupan de estudiar la influencia de los valores y las 
culturas nacionales y personales en el desempeño de los roles. En este tipo de enfoques prevalecen los diseños de investigación correlacionales o explicativos, que permiten observar la relación entre variables.

En este tipo de estudios se encuentra el 9,1\% de los artículos de la muestra de esta revisión. En especial, predominan los enfoques que atienden la influencia de los valores de la cultura nacional en el desempeño del rol (Kiambi \& Nadler, 2012; Kim \& Kim, 2010; Vasquez \& Taylor, 1999) y los artículos que enfocan la incidencia de aspectos axiológicos y éticos, personales o institucionales, en el desempeño del rol (Han, Hyun, \& Jeong, 2013; Kang, 2010).

Algunos artículos de este segmento exploran las relaciones entre el desempeño del rol profesional y diversas dimensiones de los estudios organizacionales, por ejemplo, la motivación, el estilo de liderazgo y el tipo de organización o tipo de estructura organizacional (Gregory, 2008; Lin \& Chaidaroon, 2016; Liu, Horsley, \& Levenshus, 2010; Shin, Park, \& Cameron, 2006; Sweep, Cameron, \& Weaver-Lariscy, 1994; Van Gorp \& Pauwels, 2007; Moreno A., Navarro, Tench, \& Zerfass, 2015).

\section{Sobre la dinámica del rol del profesional de relaciones públicas}

Finalmente, los estudios sobre la dinámica del rol buscan identificar los procesos por los cuales se aprenden y se modifican los roles a lo largo del tiempo; las formas de comunicar, interpretar, aceptar y negociar las expectativas de un rol; estudian la socialización y la institucionalización de los roles — por ejemplo, la formación universitaria, la agremiación profesional, la regulación de una profesión. Estos enfoques requieren, por lo general, diseños de investigación diacrónicos o longitudinales para observar la evolución o los cambios de los roles.

Un 8,9\% de los artículos de la muestra revisada se enfoca sobre los contenidos y orientaciones de la formación académica y técnica para el rol (Berkowitz \& Hristodoulakis, 1999; Culbertson, 1985; Sallot, Cameron, \& Lariscy, 1997), la influencia de la institucionalización en el rol profesional a través de las universidades, asociaciones y modelos institucionalizados de roles (Krohling Kunsch \& Nassar de Oliveira, 2009; Molleda \& Athaydes, 2003; Sha, 2011) y algunos pocos artículos enfocan el aprendizaje y la socialización del rol a lo largo de la carrera del profesional (Powell \& Pieczka, 2016).

\section{Reflexión final}

En la literatura científica sobre relaciones públicas prevalecen los enfoques profesionales por sobre los teóricos, y entre aquellos, se destaca la preocupación por desarrollar modelos y tipologías de roles profesionales. 
La revisión de estos últimos muestra que predominan las tipologías de carácter deductivo que se basan en lo que los profesionales reportan sobre las actividades que desempeñan. Estas respuestas están influidas por la carrera profesional de los respondentes y son contingentes al nivel jerárquico en que se encuentra el profesional y al tipo de organización y unidad que integra. La mayoría de las tipologías no distinguen entre actividades propiamente administrativas - comunes a varios puestos en el ámbito organizacional- $\mathrm{y}$ actividades propias del rol profesional en las relaciones públicas. En particular la tipología dicotómica de gerente y técnico de comunicación no es excluyente, ya que un mismo profesional puede desempeñar actividades de ambos roles y tampoco es una tipología universal, ya que no aplica a todo tipo de organización y a todas las modalidades laborales del profesional, como, por ejemplo, al consultor. Además, en esta tipología no se incorporan actividades que pueden responder a otras competencias del rol del profesional de las relaciones públicas como las competencias políticas y las creativas.

Otros modelos de tipologías se concentran en las funciones o aportes que tiene el rol del profesional de las relaciones públicas para las organizaciones. Estas derivan de la literatura y también se elaboran a partir de las declaraciones de los profesionales.

Solo un estudio integra, además de las declaraciones de los profesionales actuantes del rol, la percepción de quienes interactúan con él, en este caso, en la relación consultor-cliente. En este sentido, una posible línea de investigación futura podría centrarse en estudiar el rol del profesional de las relaciones públicas sumando las percepciones y expectativas que tienen los diferentes actores involucrados en el desempeño de este rol: empleadores, clientes, educadores, públicos, otros profesionales, líderes de opinión, entre otros.

Asimismo, los resultados de la revisión realizada en las bases de Web of Science, muestran que la literatura científica sobre el rol del profesional de las relaciones públicas se ha concentrado en el estudio del desempeño del rol. Dentro de este enfoque prevalecen los estudios sobre las tensiones y conflictos entre el rol del profesional de las relaciones públicas y el periodista; la discriminación, ya sea en reconocimiento o remuneración según el sexo del profesional; las propuestas de tipologías de roles, en particular la aplicación en diferentes países y la discusión sobre el modelo dicotómico de Broom y Dozier, y el relevamiento de los contenidos y las competencias requeridas para el desempeño del rol del profesional de relaciones públicas.

Los estudios propios de la situación y de la dinámica del rol han sido menos investigados. En este sentido, y a modo de ejemplo, una serie de temas relevantes para continuar las investigación podrían ser: la relación del rol profesional con la construcción de autoridad y legitimidad; la relación entre las competencias requeridas para el rol y el estilo de liderazgo; la influencia del rol del profesional en el clima y la motivación 
interna; la función del rol del profesional en los procesos de cambio organizacional; los procesos de aprendizaje y de socialización de roles, ya sea desde la universidad, los ámbitos gremiales y el aprendizaje específico con la experiencia laboral.

\section{Referencias}

Algren, M., \& Eichhorn, K. C. (2007). Cognitive communication competence within public relations practitioners: Examining gender differences between technicians and managers. Public Relations Review, 33, 77-83. doi: https://doi.org/10.1016/j. pubrev.2006.11.010.

Alikilic, O., \& Atabek, U. (2012). Social media adoption among Turkish public relations professionals: A survey of practitioners. Public Relations Review, 38(1), 56-63. doi: https://doi.org/10.1016/j.pubrev.2011.11.002.

Argyris, C. (1961). Explorations in Consulting-Clients Relationships. Human Organization, 20(3), 121-133. doi: https://doi.org/10.17730/ humo.20.3.62kj82j7u834tk40

Berkowitz, D., \& Hristodoulakis, I. (1999). Practitioner Roles, Public Relations Education, and Professional Socialization: An Exploratory Study. Journal of Public Relations Research, 11(1), 91-103. doi: https://doi.org/10.1207/ s1532754xjprr1101_04.

Berkowitz, D., \& Lee, J. (2004). Media relations in Korea: Cheong between journalist and public relations practitioners. Public Relations Review, 30(4), 431-437. doi: https://doi.org/10.1016/j.pubrev.2004.08.011.

Botan, C. H., \& Taylor, M. (2004). Public Relations: State of the Field. Journal of Communication, 54(4), 645-661. doi: https://doi.org/10.1111/j.1460-2466.2004. tb02649.x.

Broom, G. M., \& Smith, G. D. (1979). Testing the Practitioners's Impact on Clients. Public Relations Review, 5(3), 47-59. doi: https://doi.org/10.1016/ S0363-8111(79)80027-2.

Broom, G., \& Dozier, D. (1986). Advancement for public relations role models. Public Relations Review, 12(1), 37-56. doi: https://doi.org/10.1016/ S0363-8111(86)80039-X.

Brown, K. A., White, C., \& Waymer, D. (2011). African-American students' perceptions of public relations education and practice: implications for minority recruitment. Public Relations Review, 4(5), 522-529. doi: https://doi.org/10.1016/j. pubrev.2011.09.017.

Callison, C., Merle, P. F., \& Seltzer, T. (2014). Smart friendly liars: Public perception of public relations practitioners over time. Public Relations Review, 40(5), 829-831. doi: https://doi.org/10.1016/j.pubrev.2014.09.003. 
Castillo Esparcia, A. (2009). Relaciones Públicas. Teoría e historia. Barcelona, España: UOC.

Chen, Y.-N. K. (2011). Social capital, human capital, and career success in public relations in Taiwan. Chinese Journal of Communication, 4(4), 430-449. doi: https://doi.org/10.1080/17544750.2011.616290.

Cutlip, S. M., \& Center, A. H. (1971). Effective Public Relations (4 ed). Englewood Cliffs, NJ: Prentice Hall.

DeSanto, B., \& Moss, D. (2005). Rediscovering what PR managers do: Rethinking the measurement of managerial behavior in the public relations context. Journal of Communication Management, 9(2), 179-196. doi: https://doi. org/10.1108/13632540510621371.

DeSanto, B., Moss, D., \& Newman, A. (2007). Building an Understanding of the Main Elements of Management in the Communication/Public Relations Context: A Study of U. S. Practitioners' Practices. J\&MC Quarterly, 84(3), 439-454. doi: https://doi.org/10.1177/107769900708400303.

Dodd, M. D., Brummette, J., \& Hazleton, V. (2015). A social capital approach: An examination of Putnam's civic engagement and public relation roles. Public Relations Review, 41(4), 472-479. doi: https://doi.org/10.1016/j.pubrev.2015.05.001.

Dozier,D.M.(1984).Program Evaluation and the roles of practitioners.Public Relations Review, 10(2), 13-21. doi: https://doi.org/10.1016/S0363-8111(84)80002-8.

Dozier, D. M. (1985). Planning and Evaluation in PR Practice. Public Relations Review, 11(2), 17-25. doi: https://doi.org/10.1016/S0363-8111(82)80115-X.

Dozier, D. M., \& Broom, G. M. (1995). Evolution of the manager role in public relations practice. Journal of Public Relations Research, 7(1), 3-26. doi: https:// doi.org/10.1207/s1532754xjprr0701_02.

Dozier, D. M., \& Broom, G. M. (2006). The Centrality of Practitioner Roles to Public Relations Theory. En C. H. Botan, \& V. Hazleton, Public Relations Theory II (pp. 137-170). New York, NY: Routledge.

Ferguson, M. A. (1984). Building theory in public relations: Interorganizational relationships as public relations paradigm. Annual Conference of the Association for Education in Journalism and Mass Communication. Gainesville, FL.

Frohlich, R., Koch, T., \& Obermaier, M. (2013). What's the harm in moonlighting? A qualitative survey on the role conflicts of freelance journalists with secondary employment in the field of PR. Media, Culture \& Society, 35(7), 809-829. doi: https://doi.org/10.1177/0163443713495076.

Gregory, A. (2008). Competencies of senior communication practitioners in the UK: An initial study. Public Relations Review, 34, 215-223. doi: https://doi. org/10.1016/j.pubrev.2008.04.005. 
Gregory, A. (2015). Practitioner-leaders' representation of roles: The Melbourne Mandate. Public Relations Review, 41(5), 598-606. doi: https://doi.org/10.1016/j. pubrev.2014.02.030.

Grunig, J. E. (1992). Excellence in public relations and communication management. Hillsdale, NJ: Lawrence Erlbaum.

Grunig, J. E., \& Huang, Y. H. (2000). From organizational effectiveness to relationship indicators: Antecedents of relationships, public relations strategies, and relationship outcomes. En J. A. Ledingham, \& S. D. Bruning, Public relations as relationship management: A relational approach to the study and practice of public relations (pp. 23-53). Hillsdale, NJ: Erlbaum.

Grunig, J. E., \& Hunt, T. (1984). Managing public relations (Dirección de relaciones públicas). New York, NJ: Holt, Rinehart \& Winston.

Guth, D. (1995). Organizational crisis experience and Public-Relations roles. Public Relations Review, 21(2), 123-136. doi: https://doi. org/10.1016/0363-8111(95)90003-9.

Ha, J. H., \& Ferguson, M. A. (2015). Perception discrepancy of public relations functions and conflict among disciplines: South Korean public relations versus marketing professionals. Journal of Public Relations Research, 27(1), 1-21. doi: https://doi.org/10.1080/1062726X.2014.924838.

Han, J. Y., Hyun, S., \& Jeong, H. (2013). Individual and organizational antecedents of professional ethics of public relations practitioners in Korea. Journal of Business Ethics, 116(3), 553-566. doi: https://doi.org/10.1007/s10551-012-1480-6.

Heath, R., Leth, S., \& Nathan, K. (1994). Communicating services quality improvement-Another role for Public-Relations. Public Relations Review, 20(1), 29-39. doi: https://doi.org/10.1016/0363-8111(94)90112-0.

Hu, Y., \& Pang, A. (2016). Public relations practitioner's perception of the use of crisis response strategies in China. Public Relations Review, 42(2), 333-335. doi: https:// doi.org/10.1016/j.pubrev.2016.02.006.

Hutton, J. (1999). The definition, dimensions and domains of public relations. Public Relations Review, 25(2), 199-214. doi: https://doi.org/10.1016/ S0363-8111(99)80162-3.

Jiang, H., \& Shen, H. (2013). Toward a theory of public relations practitioners' own conflict: work versus life. Journal of Public Relations Research, 25, 259-279. doi: https://doi.org/10.1080/1062726X.2013.788446.

Kang, J.-A. (2010).Ethical conflict and job satisfaction of public relations practitioners. Public Relations Review, 36(2), 152-156. doi: https://doi.org/10.1016/j. pubrev.2009.11.001.

Kiambi, D. M., \& Nadler, M. K. (2012). Public relations in Kenya: An exploration 
of models and cultural influences. Public Relations Review, 38(3), 505-507. doi: https://doi.org/10.1016/j.pubrev.2012.01.007.

Kim, S.-Y., \& Reber, B. H. (2015). Public relations' place in corporate social responsibility: Practitioners define their role. Public Relations Review, 34(4), 337342. doi: https://doi.org/10.1016/j.pubrev.2008.07.003.

Kim, Y., \& Bae, J. (2006). Korean practitioners and journalists: Relational influences in news selection. Public Relations Review, 32(3), 241-245. doi: https://doi. org/10.1016/j.pubrev.2006.05.016.

Kim, Y., \& Kim, S.-Y. (2010). The influence of cultural values on perceptions of corporate social responsibility: Application of Hofstede's dimensions to Korean public relations practitioners. Journal of Business Ethics, 91(4), 485-500. doi: https://doi.org/10.1007/s10551-009-0095-z.

Krohling Kunsch, M. M., \& Nassar de Oliveira, P. R. (2009). The relationship between the academy and professional organizations in the development of organizational communication. Management Communication Quarterly, 22(4), 655-662. doi: https://doi.org/10.1177/0893318909332276.

L'Etang, J. (2009). Relaciones públicas: conceptos, práctica y crítica. Barcelona, España: UOC (primera edición en inglés 2008, Sage).

Lauzen, M. (1992). Public relations roles, intraorganizational power, and encroachment. Journal of Public Relations Research, 4(2), 61-80. doi: https://doi. org/10.1207/s1532754xjprr0402_01.

Le Roux, T. (2014). The description of South African corporate communication practitioners that contribute to organisational performance. Public Relations Review, 40(2), 193-215. doi: https://doi.org/10.1016/j.pubrev.2013.11.008.

Lee, N. M., Sha, B.-L., Dozier, D. M., \& et al. (2015). The role of new public relations practitioners as social media experts. Public Relations Review, 41(3), 411-413. doi: https://doi.org/10.1016/j.pubrev.2015.05.002.

Leichty, G., \& Springston, J. (1993). Reconsidering public relations models. Public Relations Review, 19(4), 327-339. doi: https://doi. org/10.1016/0363-8111(93)90055-H.

Leichty, G., \& Springston, J. (1996). Elaborating public relations roles. Journalism and Mass Communication Quaterly, 73(2), 467-477. doi: https://doi. org/10.1177/107769909607300215.

Li, C., Cropp, F., Sims, W., \& Jin, Y. (2012). Perceived professional standards and roles of public relations in China: Through the lens of Chinese public relations practitioners. Public Relations Review, 38(5), 704-710. doi: https://doi. org/10.1016/j.pubrev.2012.05.001.

Lin, S., \& Chaidaroon, S. (2016). Developing a Preliminary Model of Situational PR 
Leadership: Empirical Evidence from Singapore. Asia Pacific Public Relations Journal, 17(2), 28-45. Recuperado de http://novaojs.newcastle.edu.au/apprj/ index.php/apprj/article/view/79.

Liu, B. F., Horsley, J. S., \& Levenshus, A. B. (2010). Government and Corporate Communication Practices: Do the Differences Matter? Journal of Applied Communication Research, 38(2), 189-213. doi: https://doi. org/10.1080/00909881003639528.

Macnamara, J. (2016). The continuing Convergence of Journalism and PR: New Insights for Ethical Practice From a Three-Country Study of Senior Practitioners. Journalism \& Mass Communication Quarterly, 93(1), 118-141. doi: https://doi. org/10.1177/1077699015605803.

Mellado, C., \& Barría, S. (2012). Development of professional roles in the practice of public relations in Chile. Public Relations Review, 38(3), 446-453. doi: https://doi. org/10.1016/j.pubrev.2012.04.001.

Mellado, C., \& Hanusch, F. (2011). Comparing professional identities, attitudes, and views in public communication: A study of Chilean journalists and public relations practitioners. Public Relations Review, 37(4), 384-391. doi: https://doi. org/10.1016/j.pubrev.2011.08.014.

Molleda, J., \& Athaydes, A. (2003). Public Relations licensing in Brazil: evolution and the views of professionals. Public Relations Review, 29(3), 271-279. doi: https:// doi.org/10.1016/S0363-8111(03)00045-6.

Molleda, J.-C., \& Ferguson, M. A. (2004). Public Relations Roles in Brazil: Hierarchy Eclipses Gender Differences. Journal of Public Relations Research, 16(4), 327-351. doi: https://doi.org/10.1207/s1532754xjprr1604_1.

Moreno, A., Navarro, C., Tench, R., \& Zerfass, A. (2015). Does social media usage matter? An analysis of online practices and digital media perceptions of communication practitioners in Europe. Public Relations Review, 41(2), 242-253. doi: https://doi.org/10.1016/j.pubrev.2014.12.006.

Moss, D., \& Green, R. (2002). Re-examining the manager's role in public relations: What management and public relations research. Journal of Communication Management, 6(2), 118-132. doi: https://doi.org/10.1108/13632540210806982.

Moss, D., Newman, A., \& DeSanto, B. (2005). What Do Communication Managers Do? Defining and Refining the Core Elements of Management in Public Relations/ Corporate Communication Context. Jઐ MC Quarterly, 8(4), 873-890. doi: https:// doi.org/10.1177/107769900508200408.

Moss, D.,Warnaby, G., \& Newman,A. (2000).Public relations practitioner role enactment at the senior management level within U.K. companies. Journal of Public Relations Research, 12(4), 277-307. doi: https://doi.org/10.1207/S1532754XJPRR1204_1. 
Napoli, P., Taylor, M., \& Powers, G. (1999). Writing activities of public relations practitioners: The relationship between experience and writing tasks. Public Relations Review, 25(3), 369-380. doi: https://doi.org/10.1016/ S0363-8111(99)00024-7.

Neijins, P., \& Smit, E. (2006). Dutch public relations practitioners and journalists: Antagonists no more. Public Relations Review, 32(3), 232-240. doi: https://doi. org/10.1016/j.pubrev.2006.05.015.

Obermaier, M., \& Koch, T. (2014). Mind the gap: Consequences of inter-role conflicts of freelance journalists with secondary employment in the field of public relations. Journalism, 16(5), 615-629. doi: https://doi.org/10.1177/1464884914528142.

Penning, T., \& Sweetser, K. (2015). Role enactment, employer type, and pursuit of APR. Public Relations Review, 41, 135-137. doi: https://doi.org/10.1016/j. pubrev.2014.11.016.

Porter, L. V., \& Shallot, L. M. (2003). The Internet and public relations: investigating practitioners' roles and World Wide Web use. Journalism \& Mass Communication Quarterly, 80(3), 603-622. doi: https://doi.org/10.1177/107769900308000308.

Powell, M., \& Pieczka, M. (2016). Understanding learning in senior public relations practices. From boundary spanning to boundary dwelling. Journal of Communication Management, 20(4), 312-327. doi: https://doi.org/10.1108/ JCOM-11-2015-0093.

Sallot, L. M., Porter, L., \& Acosta-Alzuru, C. (2004). Practitioners' web use and perceptions of their own roles and power: A qualitative study. Public Relations Review, 30(3), 269-278. doi: https://doi.org/10.1016/j.pubrev.2004.05.002.

Sallot, L., Cameron, G., \& Lariscy, R. (1997). Professional standards in public relations: A survey of educators. Public Relations Review, 23(3), 194-216. doi: https://doi. org/10.1016/S0363-8111(97)90032-1.

Schein, E. H. (1969). Process consultation: It's role in organization development. Reading, MA: Addison-Wesley.

Sha, B., \& Toth, E. (2005). Future professionals' perceptions of work, life, and gender issues in public relations. Public Relations Review, 31(1), 93-99. doi: https://doi. org/10.1016/j.pubrev.2004.09.004.

Sha, B.-L. (2011). Accredited vs. non-accredited: The polarization of practitioners in the public relations profession. Public Relations Review, 37(2), 121-128. doi: https://doi.org/10.1016/j.pubrev.2011.02.003.

Shin, J., \& Cameron, G. (2004). Conflict measurements: analysis of simultaneous inclusion in roles, values, independence, attitudes, and dyadic adjustment. Public Relations Review, 30, 401-410. doi: https://doi.org/10.1016/j.pubrev.2004.08.001. Shin, J., Park, J., \& Cameron, G. (2006). Contingent factors: Modeling generic public 
relations practice in South Korea. Public Relations Review, 32(2), 184-185. doi: https://doi.org/10.1016/j.pubrev.2006.02.015.

Shin, J.-H., Lee, J., \& Park, J. (2012). Perceptual dynamics of pluralistic ignorance and social distance: public relations practitioners and journalists in South Korea. Asian Journal of Communication, 22(1), 19-43. doi: https://doi.org/10.1080/012 92986.2011 .622773$.

Spicer, C. (1991). Communication functions performed by public relations and marketing Practitioners. Public Relations Review, 17(3), 293-305. doi: https://doi. org/10.1016/0363-8111(91)90024-F.

Supa, D. W. (2016). Do you see what I see? An examination of perceptions between advertising and public relations professionals. Public Relations Review, 42(3), 408-417. doi: https://doi.org/10.1016/j.pubrev.2016.02.007.

Sweep, D., Cameron, G. T., \& Weaver-Lariscy, R. (1994). Rethinking constraints on public relations practice. Public Relations Review, 20(4), 319-331. doi: https://doi. org/10.1016/0363-8111(94)90093-0.

Todd, V. (2014). Public relations supervisors and Millennial entry-level practitioners rate entry-level job skills and professional characteristics. Public Relations Review, 40(5), 789-797. doi: https://doi.org/10.1016/j.pubrev.2014.05.002.

Toledano, M. (2010). Professional competition and cooperation in the digital age: A pilot study of New Zealand practitioners. Public Relations Review, 36(3), 230237. doi: https://doi.org/10.1016/j.pubrev.2010.04.009.

Toth, E.L. (1988). Making peace with gender issues in public relations. Public Relations Review, 14(3), 36-47. doi: https://doi.org/10.1016/S0363-8111(88)80046-8.

Toth, E., Serini, S., Wright, D., \& Emig, A. (1998). Trends in public relations roles: 1990-1995. Public Relations Review, 24(2), 145-163. doi: https://doi.org/10.1016/ S0363-8111(99)80048-4.

Ulloa, C., Apolo, D., \& Villalobos, J. (2015). Aproximación conceptual a la comunicación corporativa: retos y propuestas. Austral Comunicación, 4(2), 287-301. Recuperado de http://www.austral.edu.ar/ojs/index.php/australcomunicacion/article/view/137.

Van Gorp, B., \& Pauwels, L. (2007). Positioning and role of public relations in large Belgian organizations. Public Relations Review, 33(3), 301-305. doi: https://doi. org/10.1016/j.pubrev.2007.05.010.

Van Ruler, B. (2004). The communication grid: an introduction of a model of four communication strategies. Public Relations Review, 30(2), 123-143. doi: https:// doi.org/10.1016/j.pubrev.2004.01.002.

Van Ruler, B., Verčič, D., Bütschi, G., \& Flodin, B. (2001). On the definition of public relations: a European view. Public Relations Review, 27(4), 373-387. doi: https:// doi.org/10.1016/S0363-8111(01)00095-9. 
Van Ruler, B., Verčič, D., Bütschi, G., \& Flodin, B. (2004). A first look for parameters of public relations in Europe. Journal of Public Relations Research, 16(1), 35-63. doi: https://doi.org/10.1207/s1532754xjprr1601_2.

Vasquez, G., \& Taylor, M. (1999). What cultural values influence American public relations practitioners? Public Relations Review, 25(4), 433-449. doi: https://doi. org/10.1016/S0363-8111(99)00030-2.

Verčič, A. T., \& Colić, V. (2016). Journalists and public relations specialists: A coorientational analysis. Public Relations Review, 42(4), 522-529. doi: https://doi. org/10.1016/j.pubrev.2016.03.007.

Vieira Jr, E. T., \& Grantham, S. (2014). Defining public relations roles in the U.S.A. using cluster analysis. Public Relations Review, 40(1), 60-68. doi: https://doi. org/10.1016/j.pubrev.2013.11.021.

Wolf, K. (2016).Diversity in Australian Public Relations: an exploration of practitioner perspectives. Asia Pacific Public Relations Journal, 17(2), 62-77. Recuperado de http://novaojs.newcastle.edu.au/apprj/index.php/apprj/article/view/66.

Xifra, J. (2011). Manual de Relaciones Públicas e institucionales. Madrid, España: Tecnos.

Yaxley, H. (2013). Career experiences of women in British public relations (19701989). Public Relations Review, 39(2), 156-165. doi: https://doi.org/10.1016/j. pubrev.2013.03.009.

Yeo, S. L., \& Sriramesh, K. (2009). Adding value to organizations: An examination of the role of senior public relations practitioners in Singapore. Public Relations Review, 35(4), 422-425. doi: https://doi.org/10.1016/j.pubrev.2009.08.003. 